\title{
Ureteral herniation in kidney transplant - Case Report
}

\author{
Monteiro $\mathrm{LMC}^{1 *}$, Ximenes $\mathrm{SF}^{1}$, Aguiar WF ${ }^{1}$, Tedesco-Silva $\mathrm{H}^{2}$ and Medina-Pestana $\mathrm{J}^{2}$ \\ ${ }^{1}$ Division of Urology, Hospital do Rim, Universidade Federal de São Paulo, Brazil \\ ${ }^{2}$ Division of Nephrology, Hospital do Rim, Universidade Federal de São Paulo, Brazil
}

\section{Introduction}

Ureteral obstruction may occur any time after kidney transplantation. Donor and recipient underlying conditions and surgical complications are the predominant risk factors associated with this condition. While ureteral stenosis may account for most of the ureteral obstructions diagnosed after kidney transplantation, urolithiasis and ureter herniation are less frequent etiologies. This is a case report of two rare cases regarding inguinal herniation of a transplanted ureter causing ureteral obstruction and acute kidney allograft dysfunction.

\section{Case 1}

A 67 years old male patient presented at a routine outpatient visit complaining of a gradual enlargement of an inguinal protuberance over the last six months. Prior medical history included endstage chronic kidney disease secondary to hypertension. He had received a living donor kidney transplant in 2003 with extravesical ureteroneocystostomy. The early postoperative course was uneventful and achieved optimum serum creatinine of $1,52 \mathrm{mg} / \mathrm{dL}$, receiving tacrolimus, azathioprine and prednisone.

Although there were no other symptoms, a slow increase in serum creatinine, from $1,5 \mathrm{mg} / \mathrm{dL}$ to $2,4 \mathrm{mg} / \mathrm{dL}$, was noticed in the first three post-operative years. Physical examination found a right sided inguinal hernia. Abdominal ultrasound revealed a severe hydronephrosis of the transplant kidney but the ureter could not be visualized. An abdominal computed tomography (CT) showed severe ureterohydronephrosis with herniation of the ureter into the inguinal canal (Figure 1), prompting surgical exploration. Through the previous right-side Gibson incision, the spermatic funiculi and hernial sac were identified and isolated. Exploration of the herniary sac revealed a long ureter, with a dilated proximal portion $(3 \mathrm{~cm}$ wide), followed by an abrupt transition to a rather typical distal segment, close to the bladder wall (Figure 2). Both segments appeared well vascularized and viable. Given these findings and the excessive length of the ureter, ureterectomy of the distal segment followed by a tailored ureteroplasty (Figure 3) and new Lich-Gregoir ureteroneocystostomy of the proximal segment over a double J stent was performed. The posterior wall defect was mended through Lichtenstein technique. A bladder catheter was kept for one week and the ureteral stent for one month. After the removal of the bladder catheter, ultrasound and CT scan were unremarkable (Figure 4) and serum creatinine decreased from $2.4 \mathrm{mg} / \mathrm{dL}$ to $2.05 \mathrm{mg} / \mathrm{dL}$. Patient is still in follow-up, without any sign of recurrence.

\section{Case 2}

A 50 years old male patient presented at a routine outpatient visit complaining of a gradual enlargement of an inguinal protuberance over the last four months.

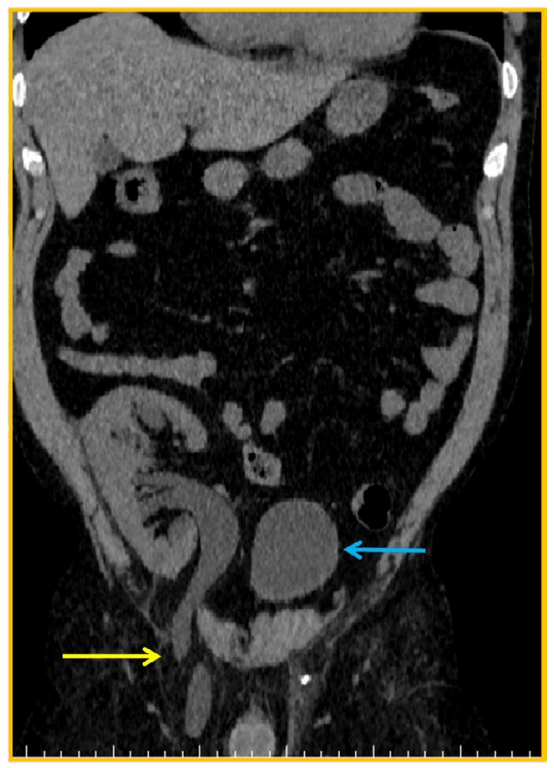

Figure 1. CT scan: blue arrow - bladder; yellow arrow - herniated ureter through the inguinal canal

Prior medical history included hypertension, obesity, smoking and end-stage chronic kidney disease secondary to acute glomerulonephritis. He received a living donor kidney transplant in 1998 with extra vesical ureteroneocystostomy. The early postoperative course was uneventful and achieved optimum serum creatinine of $1,57 \mathrm{mg} / \mathrm{dL}$.

A continuing increase in serum creatinine, from $1,5 \mathrm{mg} / \mathrm{dL}$ to $2,4 \mathrm{mg} / \mathrm{dL}$, was noticed in the first three post-operative years, but he was being treated as a case of graft chronic dysfunction. This may have contributed to the delay on the anatomical diagnosis. Physical examination revealed a right sided inguinal hernia. The ultrasound showed severe hydronephrosis, but was unable to visualize the ureter. CT scan confirmed a severe ureterohydronephrosis of the transplanted kidney with entrapment of the ureter through the inguinal canal.

It was mandatory to repair the hernia due to patient discomfort, despite debatable renal function improvement that a surgical procedure could bring to this patient. By using previous Gibson incision the spermatic funiculi and herniary sac were separated (Figure 5). The

*Correspondence to: Monteiro LMC, M.D, Division of Urology, Hospital do Rim, São Paulo, Brazil, E-mail: lilahcarvas@gmail.com

Received: August 12, 2020; Accepted: August 25, 2020; Published: August 28, 2020 


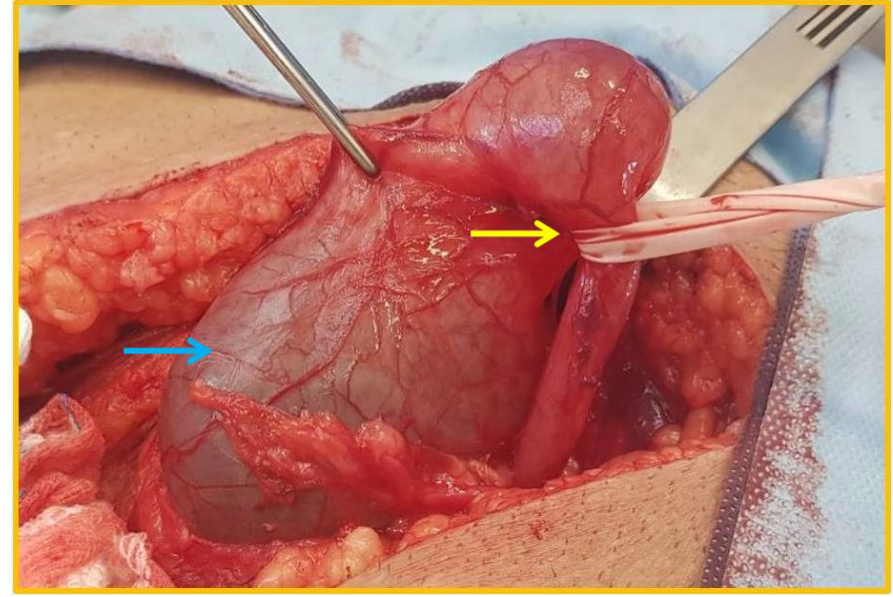

Figure 2. Blue arrow: proximal dilated portion of the transplant's ureter; yellow arrow: point of strangulation at the distal portion of the ureter

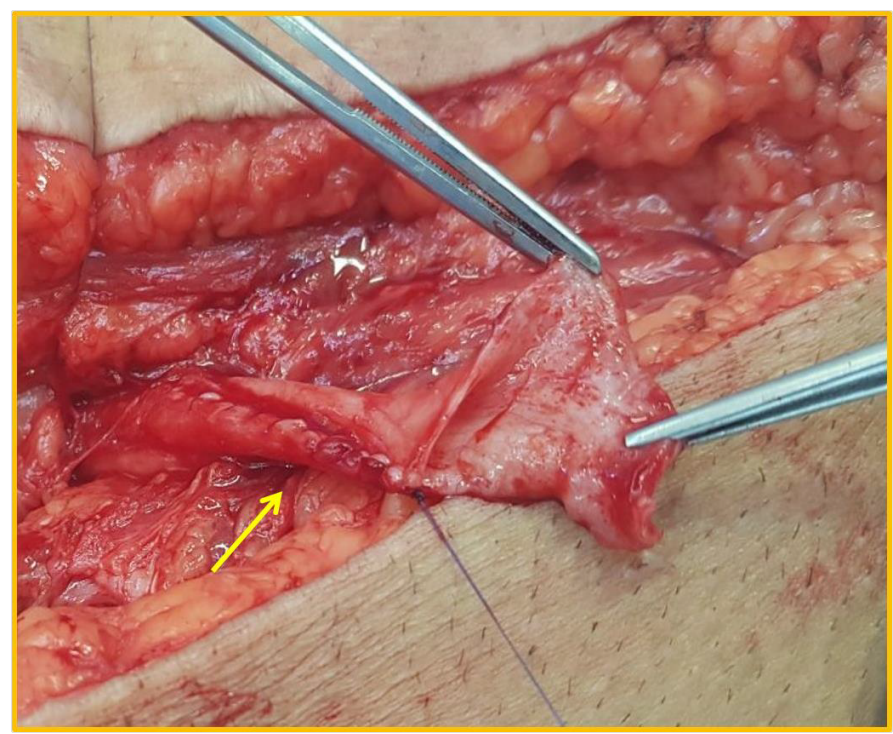

Figure 3. Tailored ureteroplasty - yellow arrow: running suture

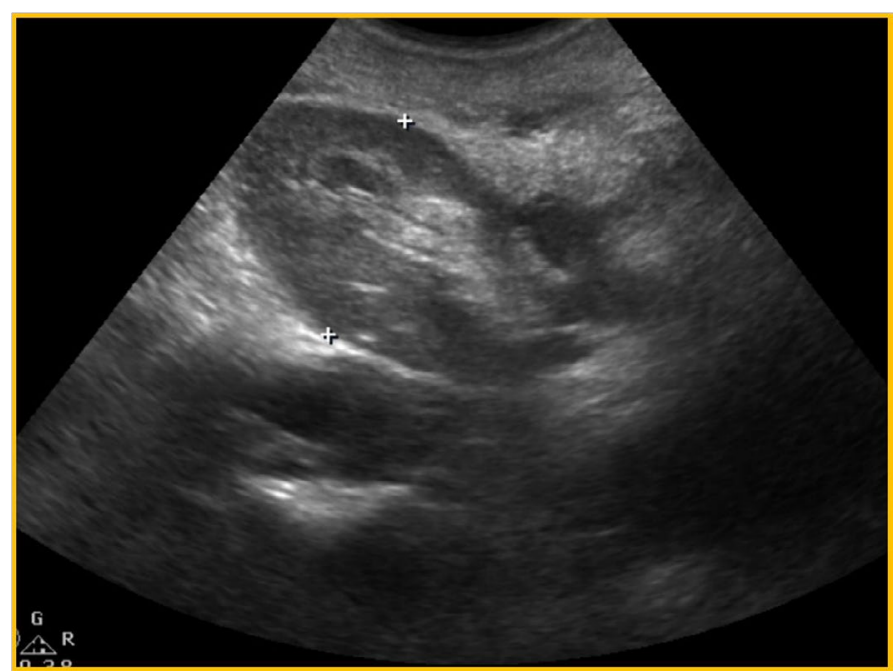

Figure 4. Post-operative ultrasound - no graft dilation

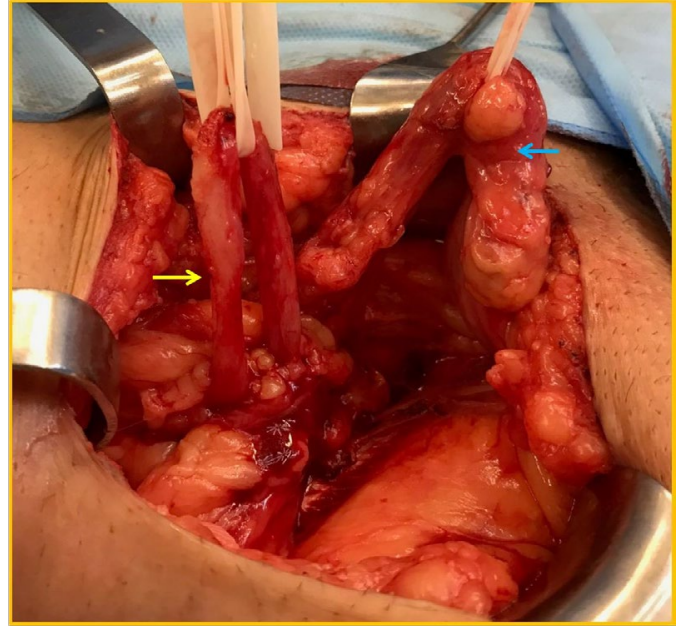

Figure 5. Blue arrow: spermatic funiculi; yellow arrow: transplant's ureter

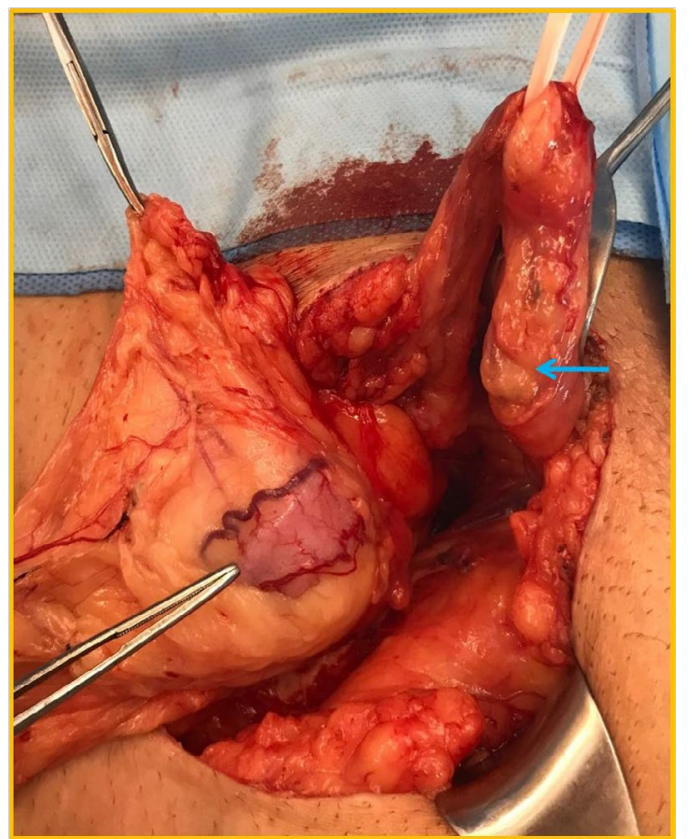

Figure 6. Tip of tweezer: ureter inside the herniary sac; blue arrow: spermatic funiculi

hernia contained part of the graft's ureter inside (Figure 6), with a moderate dilation of its proximal segment. A partial ureterectomy, followed by Lich-Gregoir re-implant was performed. No tailoring was needed in this case. The posterior wall was also repaired using the Lichtenstein technique.

Post-operative ultrasound showed there was no longer dilation of the graft. Different from CASE 1, the creatinine levels had minimum improvement, from $6,8 \mathrm{mg} / \mathrm{dL}$ to $5,7 \mathrm{mg} / \mathrm{dL}$.

\section{Discussion}

Transplant ureter inguinal hernia, causing post renal obstruction, is a rare condition, with 25 cases reported in literature. As the number of kidney transplants have grown since the 1950's, new and more worrisome post-operative complications have been diagnosed. Raising awareness for this condition is paramount in order to prevent graft 
function loss and iatrogenic lesions during exploratory operations on the inguinal canal.

This report and literature review gathers data in order to find possible factors associated to this illness, regarding specific clinical features, unique images, different clinical and surgical approaches, and kidney function follow up.

There is still no definite reason for the development of this hernia. Two possible factors related to the surgical technique are mentioned: redundant ureter and the positioning of the ureter over the spermatic funiculi $[1,2]$. Supposedly, this redundancy could enable its herniation since the ureter would be longer and closer to the inguinal canal. In a similar mechanism, the spermatic funiculi could push the ureter onto the inguinal canal, enabling its herniation.

Other possible risk factors include male sex, age 50 years or greater, obesity and having a kidney transplant for over 5 years [3].

Most of the patients had over five years of kidney transplant [3-23], and almost half of them had over ten years of the procedure [5-7,11,13,15$17,20,21,23]$. Literature does not provide any statistical evidence for the connection between post- operative time and the identification of ureteric-inguinal hernia. Nevertheless, one could infer that the chronic exposure to certain immunosuppressant could contribute to this illness, as they may interfere with part of the wound healing process. Among the main clinical features related to this anatomic pathology are previous inguinal surgery, inguinal enlargement, and acute loss of graft function $[2,3,8-10,12,14,16,17,20,21,23]$.

The main image methods used for the diagnosis are graft ultrasound, abdominal CT, and nefrography [5-11,16-17,21-23]. In five cases, abdominal magnetic resonance was used for the documentation $[3,4,9,12,19]$.

The US mostly reveals severe hydronephrosis and no visualization of ureter's full length. The CT scan shows the transplanted ureter dilation and the ureteric- inguinal hernia, making it possible to come to the anatomical diagnosis $[1,15,18]$. There was no explanation for the need of MRI, but one could think that the pelvic area and the inguinal canal anatomical features may become even more striking through these images.

Over half of the cases describe the positioning of a nephrostomy tube before any surgery in order to prevent any more graft loss [1$11,14-16,17]$. A pyelography through the nephrostomy tube could allow a refined documentation of the ureterohydronephrosis and of the transplant ureter entrapment in the inguinal canal, even though this procedure would not change clinical or surgical outcome.

One report describes that the hernia repair could not be performed, because the patient's clinical conditions did not allow any surgical procedure [11]. Eight reports showed a need for partial ureterectomy, followed by ureteral reimplantation and hernia repair [14-17,19,23]. Ureteral tailoring was performed only in one case [23]. In two cases a dilation of the ureteroneocystostomy was needed, followed by the implantation of double J stent $[6,11]$.

In eight cases the hernia repair was performed without the need of any stenting, since the patients were in good clinical conditions and were already cleared for this type of surgery $[9,12,17,18-20,23]$.

Graft function follow up was provided in most of the cases [1$3,5,6,8-10,12,14-19,21-23]$. Improvement in graft function was seen in all of them.

\section{Conclusion}

This case reports and literature review gathers the main findings about ureteric-inguinal hernia in kidney transplant patients. It provides key information about clinical features, diagnostic images, surgical treatments and graft function follow up. This article can not only be used as a guide for kidney transplant surgeons, but also as an alert to general surgeons that may encounter themselves with a case of hernia repair, in which during the inventory of the herniary sac a tubular structure is found. By knowing about this condition, they could avoid a complex iatrogenic lesion to a transplanted ureter.

\section{References}

1. Ciancio G, Burke GW, Nery J, Huson H, Coker D, et al. (1995) Positional obstructive uropathy secondary to ureteroneocystostomy herniation in a renal transplant recipient. Journal of Urology 154: 1471-1472.

2. Weingarten KE, DÁgostino HB, Dunn J, Steiner RW (1996) Obturator herniation of the ureter in a renal transplant recipient causing hydronephrosis: perioperative percutaneous management. JVIR 7: 939-941.

3. Osman Y, Ali-El-Dein B, El-Leithy R, Shokeir A (2004) Sliding hernia containing the ureter-a rare cause of graft hydroureteronephrosis: a case report. Transplantation Proceedings 36: 1402-1404.

4. Sanchez AS, Tebar JC, Martin MS, Bachs JM, Moreno MJ, et al. (2005) Obstructive uropathy secondary to ureteral herniation in a pediatric en bloc renal graft. American Journal of Transplantation 5: 2074-2077.

5. Ingber MS, Girdler BJ, Moy JF, Frikker MJ, Hollander JB (2007) Inguinal herniation of a transplant ureter: rare cause of obstructive uropathy. Urology 70: 1224.e1-e3.

6. Azhar R, Boutros M, Hassanain M, Plyhronopoulos G, Chaudhury P, et al. (2009) A rare case of obstructive uropathy in renal transplantation: ipsilateral indirect inguinal herniation of a transplant ureter. Transplantation 88: 1038-1039.

7. Odisho AY, Freise CE, Tomlanovich SJ, Vagef PA (2010) Inguinal herniation of a transplant ureter. Kidney International 78: 115.

8. Tran D, Gaboriault J (2011) Obstructive uropathy caused by an inguinal hernia in a kidney transplant recipient: report of hernia cure by the shouldice technique. Dialysis and Transplantation 40: 413-414.

9. Mukha RP, Devasia A, Thomas EM (2011) Ureteral herniation with intermitent obstructive uropathy in a renal allograft recipient. Urology Journal 70: 1224.e1-e3.

10. Youssef F, Brown P, Tappenden J, Hall J, Salim F, Shrestha B (2013) Obstructive uropathy secondary to incisional herniation of a transplant ureter - case report and review of literature. Annals of Transplantation 18: 53-56.

11. Pourafkari M, Ghofrani M, Riahl M (2013) Inguinal herniation of a transplant kidney ureter: a case report. Iran J Radiol 10: 48-50. [Crossref]

12. Vyas S, Chabra N, Singh S, Khandelwal N (2014) Inguinal herniation of the bladder and ureter: an unusual cause of obstructive uropathy in a transplant kidney. Saudi Journal of Kidney Diseases and Transplantation 25: 153-155.

13. Kondo A, Nishizawa Y, Akamoto S, Fujiwara M, Okano K, et al. (2015) Internal inguinal hernia on the transplant side after kidney transplantation: a case report. Surgical Case Reports.

14. Hakeem AR, Gopalakrishnan P, Dooldeniya MD, Irving HC, Ahmad N (2016) Inguinal Herniation of a Transplant Ureter: Lessons Learned From a Case of "Water Over the Bridge". Experimental and Clinical Transplantation 1: 103-105.

15. Anderson E, Corcoran A (2015) Obstructive Uropathy Due to an IncarceratedUreteroinguinal Hernia. World J Nephrol Urol 4: 237-239.

16. Esposito M, Ratnasekera A (2015) Sebastian E, Youssef N. Femoral herniation of transplanted ureter after deceased-donor kidney transplantation. Int J Surg Case Rep 10: 115-117. [Crossref]

17. Cheung F, Debartolo MM, Cobertino LM., Szafran AA, Estrada CC, et al. (2016) Different management options for transplant uretereal obstructions within an inguinal hernia. Case Rep Transplant 2016: 4730494. [Crossref]

18. Veroux M, Zerbo D, Ardita V, Veroux P (2016) First case report of acute renal failure after mesh-plug inguinal hernia repair in a kidney transplant recipient. Medicine 95: e3199. [Crossref] 
19. Soleymanian T, Kalantarian T, Radmard A (2016) Report of a kidney recipient with inguinal herniation of the transplant ureter. Saudi Journal of Kidney Diseases and Transplantation 27: 614-616.

20. Vigo V, Rossi L, Lisi P, Antonelli M, Lomonte C, et al. (2016) An unusual cause of ureteral obstruction in kidney transplant. GIN.

21. Koppula R, Haskal Z (2016) Reduction of inguinal hernia ureteral entrapment. JVIR 28: 920 .
22. Clark EG, Ali SN, Bugeja A, Sood MM (2017) As in real estate, location is what matters: a case report of transplant ureteral obstruction due to an inguinal hernia. Canadian Journal of Kidney Health and Disease.

23. Shihab F, Qazi Y, Mulgaonkar S, McCague K, Patel D, et al. (2017) Association of clinical events with everolimus exposure in kidney transplant patients receiving low doses of tacrolimus. American Journal of Transplantation 17: 2363-2371.

Copyright: (C2020 Monteiro LMC. This is an open-access article distributed under the terms of the Creative Commons Attribution License, which permits unrestricted use, distribution, and reproduction in any medium, provided the original author and source are credited. 